\title{
Reintroducing Anisotropic Interactions in Magic-Angle-Spinning NMR of Half- Integer Quadrupolar Nuclei: 3D MQMAS ${ }^{\dagger}$
}

\author{
Sungsool Wi, Henrike Heise, and Alexander Pines* \\ Materials Sciences Division, Lawrence Berkeley National Laboratory and Department of Chemistry, University of \\ California at Berkeley, Berkeley, CA 94720,
}

RECEIVED DATE (will be automatically inserted after manuscript is accepted)

The majority $(72 \%)$ of the magnetically active nuclides in the Periodic Table possess half-integer spins higher than $1 .{ }^{1}$ Hence solid-state NMR methods that provide not only sitespecific resolution but also dipolar coupling and chemical shift information of half-integer quadrupolar nuclei are enormously useful to fully characterize the structural properties. Highresolution spectra of half-integer quadrupolar nuclei can be obtained by averaging away the second-order quadrupolar broadening by means of double rotation (DOR), ${ }^{2}$ dynamic angle spinning (DAS), ${ }^{3}$ multiple-quantum magic angle spinning (MQMAS) $^{4}$, or satellite-transition magic angle spinning (STMAS). ${ }^{5}$ MQMAS is the most widely used method due to its technical simplicity. The use of MAS, however, nullifies the second-rank anisotropic interactions such as the chemical shift anisotropy (CSA) and dipolar interactions. Knowledge of these would provide valuable information about the electronic environment and structural connectivity.

For a heteronuclear spin pair $I-S$ with $I=1 / 2, S \geq 3 / 2$, high-resolution NMR spectra with dipolar information can be obtained by combining the rotational echo double resonance (REDOR) technique ${ }^{6,7}$ with MQMAS. ${ }^{8}$ Dipolar recoupling in a homonuclear quadrupolar spin pair is more demanding due to the fact that the applied $r f$ nutation fields have to compete with the much larger quadrupolar frequency. An excitation of sixquantum coherence or spin diffusion in a pair of dipole coupled spins $S=3 / 2$ has been discussed in the context of MQMAS. ${ }^{9,10}$ Three dimensional (3D) dynamic-angle correlation spectroscopy (DACSY) ${ }^{11}$ yields isotropic shift, characteristic for each chemical site, as well as the second- and fourth-rank anisotropic terms, which contain the principal values and relative orientations of both the CSA and quadrupolar interactions. This method is technically demanding due to the use of two rotational angles.

In this Communication, we present a 3D experiment which recovers homonuclear dipolar (HMD) interaction and the CSA while retaining site-specific resolution by combining rotary resonance recoupling $\left(\mathrm{R}^{3}\right)^{12,13}$ with the MQMAS experiment. Homonuclear dipolar coupling can be selectively recoupled within the central transition without contributions from CSA, heteronuclear dipolar coupling, or quadrupolar coupling for half-integer quadrupolar nuclei at the rotary resonance (RR) condition $\omega_{\mathrm{rf}}=\omega_{\mathrm{r}} /(2 S+1) .{ }^{14}$ Baldus et al used mixing at the transverse mode in a $2 \mathrm{D}$ homonuclear correlation experiment that yields cross-peaks, ${ }^{14}$ whereas in our experiment longitudinal mixing is incremented in an indirect time domain yielding an explicit dipolar Pake pattern. The advantage of longitudinal mixing over transverse mixing is that only $T_{1}$ relaxation is present during the RR mixing period. A calibration experiment is required to identify the relative sizes of spinning speed and $r f$ intensity during the spin-locking of the central transition coherence (CTC) that determine the various RR conditions. ${ }^{15}$ Only double-quantum coherence terms can be reintroduced into the effective Hamiltonian at $\omega_{\mathrm{rf}}=\omega_{\mathrm{r}} / 4$. Fig. 1A shows the 3D
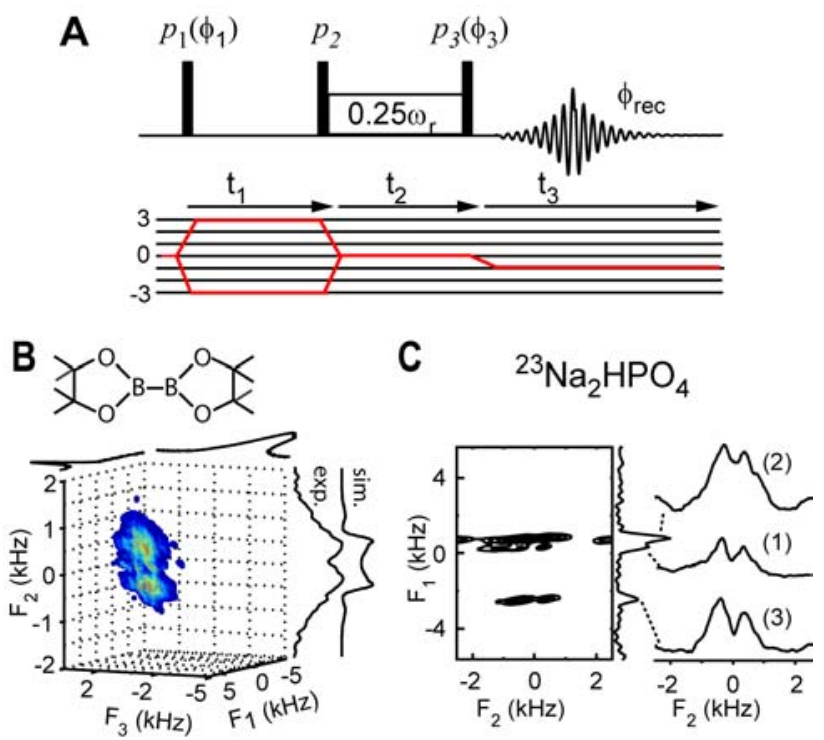

Figure 1. 3D experiment combining MQMAS with longitudinal mixing at $\omega_{\mathrm{rf}}=\omega_{\mathrm{r}} / 4$. (A) The pulse sequence used in the experiment. The phase cycling was as follows: $\phi_{1}=\left(0^{\circ}, 60^{\circ}, 120^{\circ}, 180^{\circ}, 240^{\circ}, 300^{\circ}\right)_{4} ; \phi_{3}=$ $\left(0^{\circ}\right)_{6},\left(90^{\circ}\right)_{6},\left(180^{\circ}\right)_{6},\left(270^{\circ}\right)_{6}$; $\phi_{\mathrm{rec}}=\left(0^{\circ}, 180^{\circ}\right)_{3},\left(90^{\circ}, 270^{\circ}\right)_{3},\left(180^{\circ}, 0^{\circ}\right)_{3}$, $\left(270^{\circ}, 90^{\circ}\right)_{3}$. A hypercomplex data set was obtained by collecting, for each $t_{1}$, a complementary set of 24 FID's with $\phi_{1}=\left(30^{\circ}, 90^{\circ}, 150^{\circ}, 210^{\circ}\right.$, $\left.270^{\circ}, 330^{\circ}\right)_{4}$. All the pulses were rotor synchronized; $t_{1}$ and $t_{3}$ were incremented with the rotor period $\tau_{\mathrm{r}}$ and $t_{2}$ with $4 \tau_{\mathrm{r}}$. (B) Spectrum measured for BPDB with an isolated ${ }^{11} \mathrm{~B}-{ }^{11} \mathrm{~B}$ pair. Top view onto the $F_{1^{-}}$ $F_{3}$ plane provides a normal $2 \mathrm{D}$ MQMAS spectrum and side view onto the $F_{1}-F_{2}$ plane renders the $2 \mathrm{D}$ site-specific dipolar spectrum. Both experimental and simulated dipolar spectra are compared. (C) ${ }^{23} \mathrm{Na} 3 \mathrm{D}$ dipolar $\mathrm{R}^{3}$ MQMAS measured on $\mathrm{Na}_{2} \mathrm{HPO}_{4}$. The 2D projection onto the $F_{1}-F_{2}$ plane shows site-specific dipolar coupling information for all three sites. All the spectra were taken at $11.7 \mathrm{~T}$ with a $3.2 \mathrm{~mm}$ rotor spinning at $20 \mathrm{kHz}$.

sequence applied to homonuclear dipolar recoupling for spin $3 / 2$ nuclei; the longitudinal mixing pulse is incorporated in a $z$ filtered version of the MQMAS experiment. ${ }^{16}$ The triple quantum coherence (TQC) excited by the first hard pulse evolves during $t_{1}$ and is converted into zero quantum coherence (ZQC) by another hard pulse before undergoing a RR mixing at $\omega_{\mathrm{rf}}=\omega_{\mathrm{r}} / 4$ for dipolar recoupling during $t_{2}$. Finally, the acquisition of the CTC during $t_{3}$ is carried out after applying a $\pi / 2$ detection pulse. Hypercomplex phase cycling ${ }^{17}$ was applied to $p_{1}$ together with CYCLOPS ${ }^{18}$ phase cycling at $p_{3}$ in order to give a purely absorptive line shape. Fig. 1B shows the 3D dipolar $\mathrm{R}^{3}$ MQMAS spectrum for bis(pinacolato)diboron (BPDB) with an isolated ${ }^{11} \mathrm{~B}-{ }^{11} \mathrm{~B}$ spin pair. The $2 \mathrm{D}$ projection onto the $F_{1}-F_{2}$ plane provides the dipolar Pake pattern of each site while the $2 \mathrm{D}$ projection onto the $F_{1}-F_{3}$ plane gives the usual 
MQMAS spectrum. The measured line width of the dipolar Pake pattern of the ${ }^{11} \mathrm{~B}-{ }^{11} \mathrm{~B}$ pair obtained along $F_{2}$ is $\sim 1.3 \mathrm{kHz}$. This agrees well with a simulated line shape with a dipolar coupling constant $D_{z z}$ of $2.5 \mathrm{kHz}(1.71 \AA),{ }^{19}$ scaled by the factor $3 D_{z z} \sin 2 \beta / 4 \sqrt{2}$, which is also known for double quantum recoupling in a homonuclear spin-1/2 pair, ${ }^{20}$ where $D_{z z}=$ $\left(\mu_{0} / 4 \pi\right)\left(\gamma^{2} \hbar / r^{3}\right)$ and $\beta$ is the angle relating dipolar vector to the rotor fixed frame. Fig. 1C shows the site-specific dipolar projection of a $3 \mathrm{D}$ dipolar $\mathrm{R}^{3}$ MQMAS spectrum obtained for $\mathrm{Na}_{2} \mathrm{HPO}_{4}$. The three different sodium sites of this compound are well resolved, and the homonuclear dipolar Pake patterns are evident along the $F_{2}$ dimension. The complicated network of sodium sites, however, makes a quantitative interpretation difficult. $^{14}$
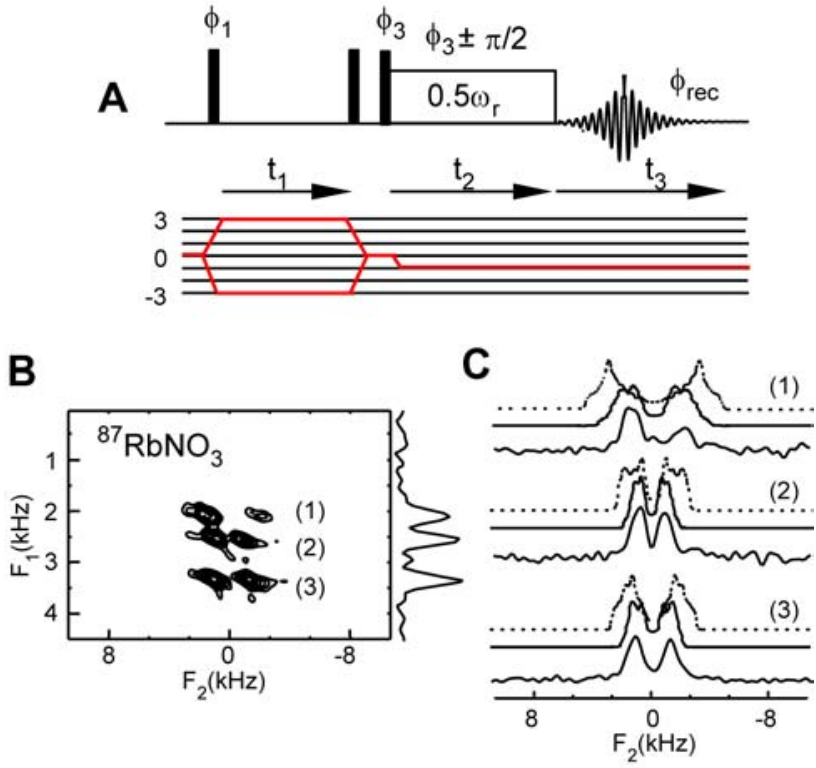

Figure 2. 3D $\mathrm{R}^{3} \mathrm{MQMAS}$ experiment with a $\mathrm{RR}$ mixing at $\omega_{\mathrm{rf}}=\omega_{\mathrm{r}} / 2$. (A) A hypercomplex acquisition was performed to provide purely absorptive peaks in the MQMAS dimension. A transverse mixing pulse with $\omega_{\mathrm{rf}}=\omega_{\mathrm{r}} / 2$ was applied during $t_{2}$ before direct NMR acquisition during $t_{3}$. The same phase cycling scheme was used as in Figure 1. (B) 2D projection onto the $F_{1}-F_{2}$ plane obtained from the 3D CSA R MQMAS spectrum of $\mathrm{RbNO}_{3} . F_{1}$ provides the isotropic spectrum, and $F_{2}$ provides CSA information. (C) Anisotropic projections for each site. Simulations with (solid line) and without (dashed line) CSA were also included. A small positive value of the CSA leads to a reduction of the line width caused by the second-order quadrupolar interaction. Inclusion of CSA explains the recoupled line width of each site (site 1: $\mathrm{C}_{\mathrm{Q}}$ $\left(\mathrm{e}^{2} \mathrm{qQ} / 2 \pi \hbar\right)=2.4 \mathrm{MHz}, \eta_{\mathrm{Q}}=0.7, \delta_{\mathrm{CSA}}=46 \mathrm{ppm}, \eta_{\mathrm{CS}}=0.4,\left\{45^{\circ}, 15^{\circ}\right.$, $\left.45^{\circ}\right\}_{\mathrm{CSA} \rightarrow \mathrm{Q}}$; site $2: \mathrm{C}_{\mathrm{Q}}=1.8 \mathrm{MHz}, \eta_{\mathrm{Q}}=0.3, \delta_{\mathrm{CSA}}=28 \mathrm{ppm}, \eta_{\mathrm{CS}}=0.6$, $\left\{0^{\circ}, 25^{\circ}, 60^{\circ}\right\}_{\mathrm{CSA} \rightarrow \mathrm{Q}}$; site $3: \mathrm{C}_{\mathrm{Q}}=1.9 \mathrm{MHz}, \eta_{\mathrm{Q}}=0.5, \delta_{\mathrm{CSA}}=31 \mathrm{ppm}, \eta_{\mathrm{CS}}$ $=0.5,\left\{90^{\circ}, 15^{\circ}, 45^{\circ}\right\}$ CSA $\rightarrow$ Q $) . .^{10}$

RR conditions defined by $\omega_{\mathrm{rf}}=n \omega_{\mathrm{r}} / 2(n=1,2)$ can also be included in the MQMAS pulse sequence. These conditions do not possess any selectivity for the recoupling of different types of anisotropic interactions such as CSA, dipolar coupling and the second-order quadrupolar interaction. The dipolar influences at these modes are, however, not usually significant compared to the size of the second-order quadrupolar interaction and CSA due to the small magnetogyric ratio and/or possibly the low natural abundance of quadrupolar nuclei involved. Moreover, the influence of the second-order quadrupolar interaction can be exactly determined based on the 1D MAS or MQMAS spectra. Therefore, the CSA can be estimated from the deviation of the line shape in the recoupled spectrum from the one expected for a pure quadrupolar interaction. Fig. 2A shows the $3 \mathrm{D} \mathrm{R}^{3} \mathrm{MQMAS}$ pulse sequence used to obtain the additional CSA information of half-integer quadrupolar nuclei. The TQC evolving during $t_{1}$ is converted to CTC and is subjected to the RR mixing at $\omega_{\mathrm{rf}}=$ $n \omega_{\mathrm{r}} / 2(n=1,2)$ during $t_{2}$ before direct detection in $t_{3}$. A $z-$ filtered MQMAS version with a hypercomplex cycling of the TQC excitation pulse was employed together with CYCLOPS phase cycling for the conversion pulse. Fig. $2 \mathrm{~B}$ shows the $2 \mathrm{D}$ projection onto the $F_{1}-F_{2}$ plane of the ${ }^{87} \mathrm{Rb} 3 \mathrm{D} C S A \mathrm{R}^{3}$ MQMAS spectrum of $\mathrm{RbNO}_{3}$ at $7.05 \mathrm{~T}$. All three Rb sites are resolved and exhibit the recoupled anisotropic line shapes. Fig. $2 \mathrm{C}$ shows the projections of each $\mathrm{Rb}$ site along the anisotropic dimension that represent recouplings of the CSA and the second-order quadrupolar interaction obtained with $\omega_{\mathrm{rf}}=\omega_{\mathrm{r}} / 2$. All dipolar interactions were ignored in the present case. Numerical simulations with (solid line) and without (dotted line) CSA for all three sites of $\mathrm{RbNO}_{3}$ are also provided in Fig. 2C (see figure caption for the tensor parameters). The CSA values and the relative orientations between CSA and quadrupolar tensors of $\mathrm{RbNO}_{3}$ from the 3D DACSY experiment ${ }^{11}$ show good agreement with our 3D $\mathrm{R}^{3}$ MQMAS measurement. Inclusion of the CSA into the simulations leads to narrower line shapes than those with only the quadrupolar interaction because a positive value of the CSA leads to a cancellation effect with respect to the second-order quadrupolar term. ${ }^{21}$

$\mathrm{R}^{3}$ incorporated in the MQMAS experiment enables the observation of homonuclear dipolar coupling and the CSA of half-integer quadrupolar nuclei with site-specific resolution. Such information is valuable in extracting distance and orientation dependent information about the structure of molecules and materials. A separate manuscript is in press which explains all of the RR conditions for anisotropic recoupling for half-integer quadrupolar nuclei. ${ }^{21}$

Acknowledgment. This work was supported by the Director, Office of Science, Office of Basic Energy Sciences, Materials Sciences Division, of the U. S. Department of Energy under Contract No. DE-AC03-76SF00098. H. H. thanks the Alexander von Humboldt Foundation for a postdoctoral fellowship.

${ }^{\dagger}$ Presented in part at the $43^{\text {rd }}$ ENC meeting (poster \# W/T 066), Asilomar, CA, April $14^{\text {th }}-19^{\text {th }} 2002$

(1) Harris, R. K.; Mann, B. E. NMR and the Periodic Table; Academic Press: New York, 1970

(2) Samoson, A.; Lipmaa, E.; Pines, A. Mol. Phys. 1988, 65, 1013

(3) Mueller, K. T.; Sun, B. Q.; Chingas, G. C.; Zwanziger, J. W.; Terao, T.; Pines, A. J. Magn. Reson. 1990, 86, 470.

(4) Frydman, L.; Harwood, J. S. J. Am. Chem. Soc. 1995, 117, 5367.

(5) Gan, Z. H. J. Am. Chem. Soc. 2000, 122, 3242.

(6) Gullion, T.; Schaefer, J. J. Magn. Reson. 1989, 81, 196.

(7) Fyfe, C. A.; Mueller, K. T.; Grodney, H.; Wong-Moon, K. C. Chem. Phys. Lett. 1992, 199, 198.

(8) Fernandez, C.; Lang, P. D.; Amoureux, J. P.; Pruski, M. J. Am. Chem. Soc. 1998, 120, 2672.

(9) Duer, M. J. Chem. Phys. Lett. 1997, 277, 167.

(10) Dowell, N. G.; Ashbrook, S. E.; McManus, J.; Wimperis, S. J. Am. Chem. Soc. 2001, 123, 8135.

(11) Medek, A.; Sachleben, J. R.; Beverwyk, P.; Frydman, L. J. Chem. Phys. 1996, 104, 5374

(12) Oas, T. G.; Griffin, R. G.; Levitt, M. H. J. Chem. Phys. 1988, 89, 692.

(13) Vosegaard, T.; Florian, P.; Massiot, D.; Grandinetti, P. J. J. Chem. Phys. 2001, 114, 4618.

(14) Baldus, M.; Rovnyak, D.; Griffin, R. G. J. Chem. Phys. 2000, 112 , 5902.

(15) Sun, W.; Stephen, J. T.; Potter, L. D.; Wu, Y. J. Magn. Reson. Ser. A 1995, 116, 181.

(16) Amoureux, J. P.; Fernandez, C.; Steuernagel S. J. Magn. Reson.. 1996, A123, 116.

(17) States, D. J.; Haberkorn, R. A.; Ruben, D. J. J. Magn. Reson. 1982, 48, 286.

(18) Hoult, D. I.; Richards, R. E. Proc. Roy. Soc. (London) 1975, A344, 311.

(19) Nöth, H.; Z. Naturforsch. 1984, B 39, 1463.

(20) Nielsen, N. C.; Bildsoe, H.; Jakobsen, H. J.; Levitt, M. H. J. Chem. Phys. 1994, 101, 1805.

(21) Wi, S.; Logan, J. W.; Sakellariou, D.; Walls, J. D.; Pines, A. J. Chem. Phys. (in press). 

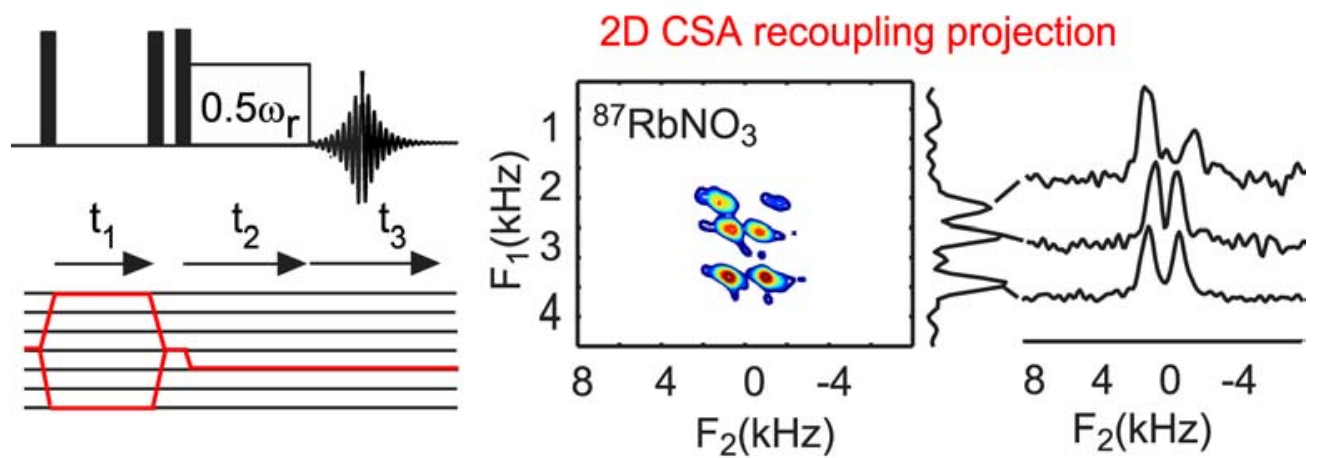

Selective reintroduction of anisotropic interactions such as the chemical shift anisotropy (CSA) and homonucler dipolar (HMD) coupling were implemented in a high-resolution NMR spectroscopy for half-integer quadrupolar nuclei. Rotary resonance recoupling $\left(R^{3}\right)$ combined with the multiple-quantum magic-angle spinning (MQMAS) in a three-dimensional (3D) experiment provides not only site-specific high-resolution spectra to yield the quadrupolar interaction parameters but also the CSA or HMD interaction parameters. This 3D experiment provides an avenue for the complete local structural information of half-integer quadrupolar nuclei. Three dimensional MQMAS experiments incorporating $\mathrm{R}^{3}$ of $\mathrm{HMD}$ and CSA interactions were demonstrated on model compounds containing ${ }^{11} \mathrm{~B},{ }^{23} \mathrm{Na}$ and ${ }^{87} \mathrm{Rb}$ nuclei. 